\title{
LO Phonon Scattering as a Depopulation Mechanism in Si/SiGe Quantum Cascades
}

\author{
P. Townsend $d^{\mathrm{a}}$, D. J. Paul ${ }^{\mathrm{a}}$, S. A. Lynch ${ }^{\mathrm{a}}$, R. W. Kelsall ${ }^{\mathrm{b}}$, Z. Ikonic ${ }^{\mathrm{b}}$, P. Harrison ${ }^{\mathrm{b}}$, D. J. Norris ${ }^{\mathrm{c}}$,

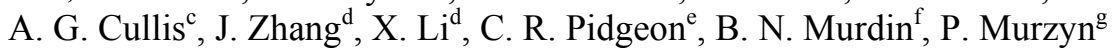 \\ ${ }^{a}$ Cavendish Laboratory, University of Cambridge, Madingley Road, Cambridge, CB3 OHE, UK \\ Telephone: +44 (0)1223 764176 Email: pt261@cam.ac.uk \\ ${ }^{\mathrm{b}}$ Institute of Microwaves and Photonics, University of Leeds, Leeds, LS2 9JT, UK \\ ${ }^{c}$ Department of Electronics and Electrical Engineering, University of Sheffield, Sheffield, S1 3JD, UK \\ ${ }^{\mathrm{d}}$ Imperial College of Science, Technology and Medicine, London, SW7 2BW, UK \\ ${ }^{\mathrm{e}}$ School of Engineering and Physical Sciences, Heriot-Watt University, Edinburgh, EH14 4AS, UK \\ ${ }^{\mathrm{f}}$ School of Electronics and Physical Sciences, University of Surrey, GU2 7XH, UK \\ ${ }^{g}$ Department of Physics, University of Sheffield, Sheffield, S3 7RH, UK
}

\begin{abstract}
The linear optical phonon as a fast depopulation mechanism in $\mathrm{Si} / \mathrm{SiGe}$ quantum cascade devices. Confirmed by pump-probe, the lifetime measurement is limited by the optical cycle of the source $(<2 \mathrm{ps})$. Fourier transform spectroscopy shows intersubband electroluminescence with good agreement to theory.
\end{abstract}

Index Terms - depopulation, laser, phonon, quantum cascade, silicon

\section{INTRODUCTION}

$\mathrm{T}$ HERE are many potential applications for terahertz radiation including medical imaging such as in dentistry and skin cancer diagnosis as well as security screening, bioweapons detection [1] and gas sensing. Such technology however, has seen limited growth due to an absence of cheap, practical sources in the far infrared. The Quantum Cascade Laser (QCL) offers one possible solution. Devices in the III-V materials system have recently demonstrated both pulsed and continuous wave emission at a number of terahertz frequencies, but are currently limited to operation below $150 \mathrm{~K}$ [2]. Realisation of a silicon heterostructure QCL could offer a number of significant advantages. The dominant, mature processing techniques available to silicon could see savings in the mass production of devices, together with the possibility of integration with current CMOS devices and the realisation of so-called system-on-chip circuits. The non-polar nature of the silicon-germanium alloy results in negligible polar optical phonon scattering. Since this temperature dependent scattering mechanism is not present, subband lifetimes are significantly enhanced and almost invariant up to $300 \mathrm{~K}$ [3] - providing great promise for devices operating at room temperature.

\section{DeVICE DESIGN AND GROWTH}

Previous work has demonstrated interwell intersubband emission from $\mathrm{Si} / \mathrm{SiGe}$ quantum cascade structures consisting of up to 100 strain-symmetrised periods at frequencies between $1.2 \mathrm{THz}(250 \mu \mathrm{m})$ and $16.2 \mathrm{THz}(18.5 \mu \mathrm{m})$ [4][5]. Population inversion is essential for lasing and typically requires fast depopulation of the lower laser level. The injector-less nature of the aforementioned interwell $\mathrm{LH}-\mathrm{HH}$ structures provided no intrinsic population inversion, as an explicit, well defined depopulationlinjection state was absent. The aim is to rectify this issue by introducing an LO phonon coupled state.

To determine this mechanism's lifetime, far infrared pump probe measurements were performed at the FELIX free electron laser facility, Holland. A three balanced beam technique was employed, with the pump and probe cross polarised in order to minimize coherence artifacts and scattered pump detection. To minimise atmospheric absorption the system was purged with dry nitrogen gas prior to measurement. A simple injector-less cascade sample, previously studied [5], was probed above and below the SiGe LO phonon energy of $62 \mathrm{meV}$. Below $62 \mathrm{meV}$ it was possible to measure the lifetime of targeted subband transitions. In this case a 30ps lifetime is measured for a $\mathrm{LH}-\mathrm{HH}$ interwell transition (Figure 1). Above $62 \mathrm{meV}$, LO phonon scattering dominates, resulting in a lifetime that is limited by the optical cycle of the pump-probe source, i.e. less than $2 \mathrm{ps}$ in this case (Figure 2). Preliminary measurements at higher beam energies suggest a sub-picosecond lifetime. 


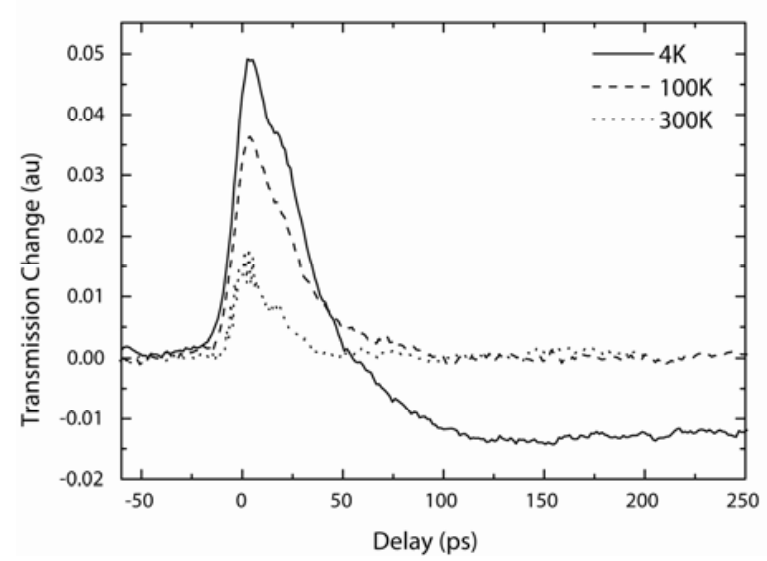

Fig. 1. The measurement of subband lifetimes below the phonon energy. An interwell transition at $14.6 \mathrm{meV}$ in an injector-less cascade is measured at $30 \mathrm{ps}$.

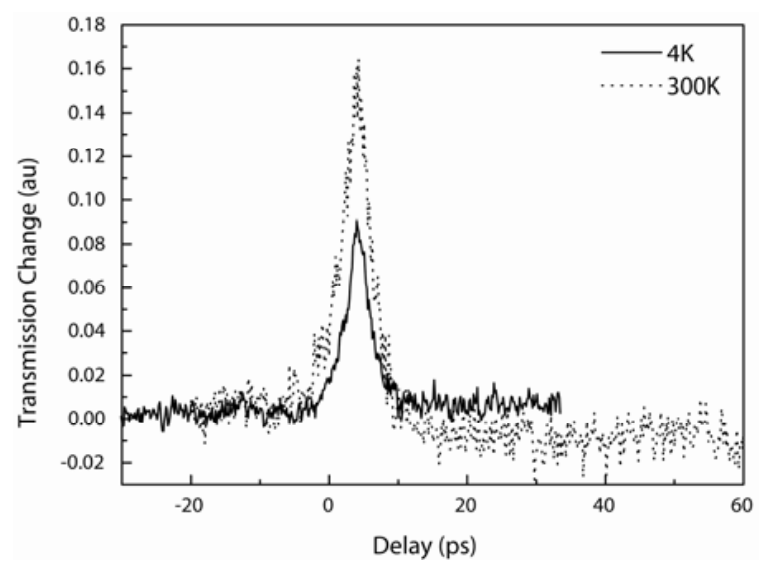

Fig. 2. Pump-probe at $73.6 \mathrm{meV}$, above the LO phonon energy, yields optical cycle limited lifetimes - less than 2 ps.

Based on this result, a new structure was designed to exploit the aforementioned phonon scattering as a means to rapidly depopulate the lower level of a quantum cascade. 6 band k.p theory was employed in engineering an appropriate band structure [6]. The radiative transition occurs between HH2 and LH1 subbands confined within the same split well (intrawell transition), with a further LO phonon coupled HH1 state separated by more than the alloy phonon energy of $62 \mathrm{meV}$, thus ensuring a fast depopulation of LH1. Figure 3 depicts one period of the 200-period strain-symmetrised structure.

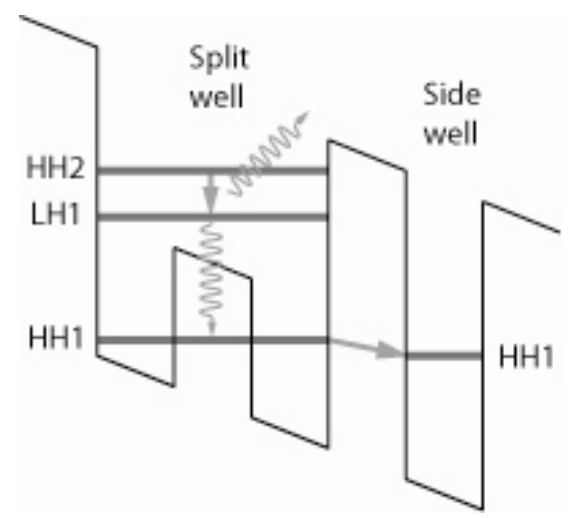

Fig. 3. Band diagram for the studied structure. The desired radiative transition occurs between HH2 and LH1. LH1-HH1 are LO phonon coupled.

The wafer was grown by gas source molecular beam epitaxy. A 2 um $\mathrm{Si}_{0.7} \mathrm{Ge}_{0.3}$ linearly graded virtual substrate and buffer layer were grown, followed by a $200 \mathrm{~nm}$ boron doped $\mathrm{Si}_{0.7} \mathrm{Ge}_{0.3}$ layer for Ohmic contacts. The active region included 200 periods consisting of a $1.93 \mathrm{~nm} \mathrm{Si}_{0.5} \mathrm{Ge}_{0.5}$ side well with $1.65 \mathrm{~nm}$ barriers either side, followed by a pair of $1.65 \mathrm{~nm}$ $\mathrm{Si}_{0.5} \mathrm{Ge}_{0.5}$ wells split by a $1.38 \mathrm{~nm} \mathrm{Si}_{0.7} \mathrm{Ge}_{0.3}$ barrier. A $10 \mathrm{~nm}$ graded injector followed by a $40 \mathrm{~nm}$ boron doped $\mathrm{Si}_{0.7} \mathrm{Ge}_{0.3}$ cap finished the structure. Cross sectional Transmission Electron Microscope (TEM) images of the wafer show excellent uniformity of the active region throughout structure (Figure 4), with threading dislocations completely confined to the virtual substrate.

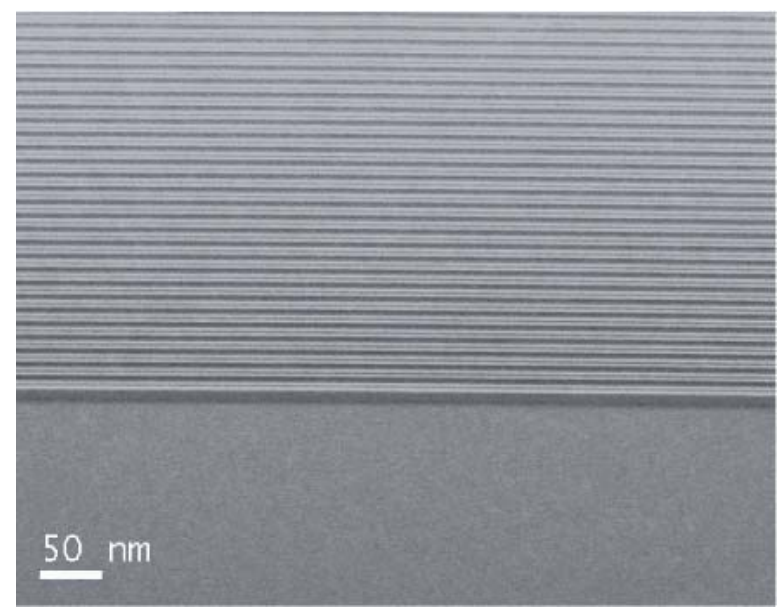

Fig. 4. Cross sectional transmission electron micrograph shows excellent uniformity of the active periods.

In order to reduce device self-heating and promote electroluminescence the wafer was processed into $200 \mu \mathrm{m}$ by $200 \mu \mathrm{m}$ square emitters. The mesa was produced by $\mathrm{CF}_{4}$ reactive ion etching, with Ohmic contacts formed by liftoffpatterned aluminium ( $1 \%$ silicon) with a rapid thermal anneal at $350^{\circ} \mathrm{C}$ for 10 minutes. To further improve heat-sinking, the devices were indium bonded to polished copper. 


\section{OPTICAL CHARACTERISATION}

The devices were optically characterised by Fourier Transform InfraRed (FTIR) spectroscopy using a Bruker 66v/S operating in step-scan mode, coupled with a liquid $\mathrm{He}$ cooled Si bolometer. Again, the optics were contained within a nitrogen atmosphere. Phase sensitive detection was used to improve signal-to-noise, accomplished by a lock-in technique gated at the detector's optimal response rate of $387 \mathrm{~Hz}(50 \%$ duty cycle), with a $50 \mathrm{kHz}$ (variable duty cycle) micropulse. Edge emission spectra are shown in Figure 5 and show good agreement with theory, Figure 6.

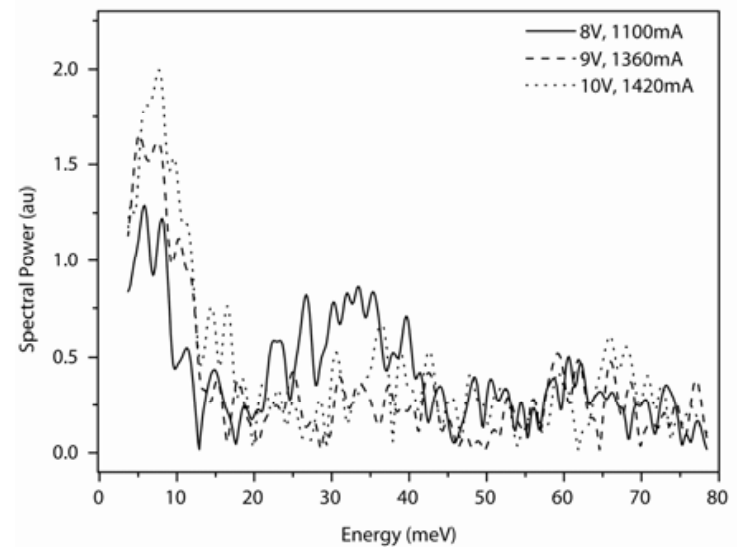

Fig. 5. Edge emitted spectra under three different biases. The peak around $8 \mathrm{meV}$ corresponds to emission from the HH2-LH1 transition.

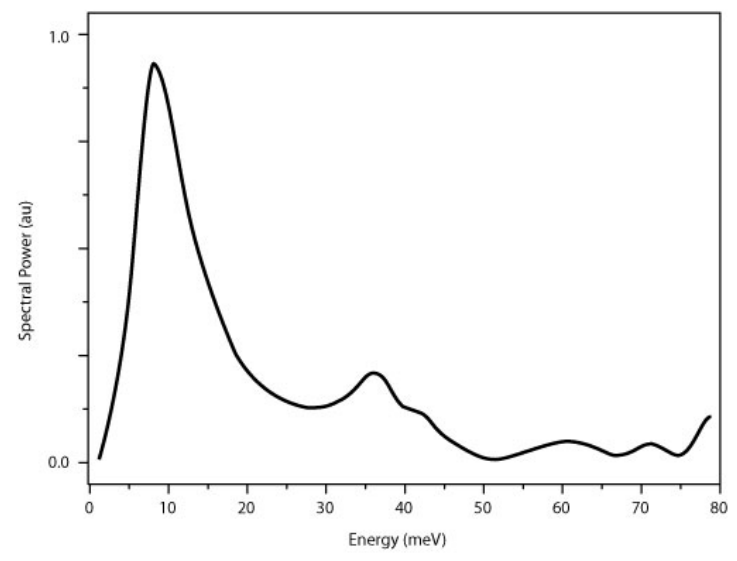

Fig. 6. Calculated edge emitted spectrum.

\section{CONCLUSION}

It has been verified that intersubband lifetimes are significantly enhanced when the superlattice is probed below the optical phonon energy. The short lifetime encountered above the phonon energy was exploited in the design of an active region that utilized this fast depopulation as a result of phonon scattering. The structure was grown and processed into suitable devices. Preliminary FTIR spectroscopy measurements demonstrate electroluminescence in good agreement with theory. Further work is required to identify lifetimes of individual states in the system and whether there is indeed population inversion in the structure.

\section{ACKNOWLEDGMENTS}

This work was supported by U.S. DARPA under Air Force Contract No. F-19628-99-C-0074 and EU research grant SHINE IST-2001-38035.

\section{REFERENCES}

[1] D. D. Arnone, C. Ciesla, M. Pepper, et al., "Terahertz imaging comes into view", Physics World, vol. 13(4), pp. 35-40, April 2000

[2] B. S. Williams, S. Kumar, H. Callebaut, et al., "Terahertz quantum cascade laser operating up to 137K", Appl. Phys. Lett., vol. 83(25), pp. 51425144, Dec. 2003.

[3] P. Murzyn, C. R. Pidgeon, C. R. Wells, et al., "Picosecond intersubband dynamics in $\mathrm{p}-\mathrm{Si} / \mathrm{SiGe}$ quantum well emitter structures", Appl. Phys. Lett., vol. 80(8), pp. 1456-1458, Feb. 2002.

[4] S. A. Lynch, R. Bates, D. J. Paul, et al., "Intersubband electroluminescence from $\mathrm{Si} / \mathrm{SiGe}$ cascade emitters at terahertz frequencies", Appl. Phys. Lett., vol. 81(9), pp. 1543-1545, Aug. 2002.

[5] R. Bates, S. A. Lynch, D. J. Paul, et al., "Interwell intersubband electroluminescence from $\mathrm{Si} / \mathrm{SiGe}$ quantum cascade emitters", Appl. Phys. Lett., vol. 83(20), pp. 4092-4094, Nov. 2003.

[6] Z. Ikonic, P. Harrison, R. W. Kelsall, "Self-consitent energy balance simulations of hole dynamics in $\mathrm{Si} / \mathrm{SiGe} \mathrm{THz}$ quantum cascade structures", $J$. Appl. Phys., vol. 96(11), pp. 6803-6811, Dec. 2004. 